\title{
The Determinant Inner Product and the Heisenberg Product of Sym(2)
}

\author{
Mircea Crasmareanu*
}

(Dedicated to the memory of Prof. Dr. Aurel BEJANCU (1946 - 2020))

\begin{abstract}
The aim of this work is to introduce and study the nondegenerate inner product $\langle\cdot, \cdot\rangle_{\text {det }}$ induced by the determinant map on the space $\operatorname{Sym}(2)$ of symmetric $2 \times 2$ real matrices. This symmetric bilinear form of index 2 defines a rational symmetric function on the pairs of rays in the plane and an associated function on the 2-torus can be expressed with the usual Hopf bundle projection $S^{3} \rightarrow S^{2}\left(\frac{1}{2}\right)$. Also, the product $\langle\cdot, \cdot\rangle_{\text {det }}$ is treated with complex numbers by using the Hopf invariant map of $\operatorname{Sym}(2)$ and this complex approach yields a Heisenberg product on $\operatorname{Sym}(2)$. Moreover, the quadratic equation of critical points for a rational Morse function of height type generates a cosymplectic structure on $\operatorname{Sym}(2)$ with the unitary matrix as associated Reeb vector and with the Reeb 1-form being half of the trace map.
\end{abstract}

Keywords: Symmetric matrix, determinant, Hopf bundle, Hopf invariant.

AMS Subject Classification (2020): Primary: 15A15 ; Secondary: 15A24; 30C10; $22 E 47$.

\section{Introduction}

This short paper, whose nature is mainly expository, is dedicated to the memory of Professor Dr. Aurel Bejancu, who was equally a gifted mathematician and a dedicated professor. As we hereby present an in memoriam paper, we feel it would be better if we choose an informal presentation rather than a classical structured discourse, where theorems and propositions are followed by their proofs.

The starting point is the remark that in order to re-prove the Herglotz lemma, in the paper [14] the 4dimensional real space $g l(2, \mathbb{R})$ is endowed with a symmetric bilinear form of index 2 (the subscript $N P$ corresponds to the initials of name of authors):

$$
<A, B>_{N P}=\frac{1}{2}[\operatorname{Tr}(A B)-\operatorname{Tr} A \cdot \operatorname{Tr} B]=\frac{1}{2}[\operatorname{det}(A-B)-\operatorname{det} A-\operatorname{det} B] .
$$

Its associated norm is:

$$
<A, A>_{N P}=-\operatorname{det} A
$$

and $\left.\operatorname{Sym}(2),<\cdot, \cdot>_{\left.N P\right|_{S y m(2)}}\right)$ is a Lorentzian 3-dimensional inner product space with the identity matrix as unit timelike vector.

The purpose of this note is to define directly on $\operatorname{Sym}(2)$ a symmetric bilinear form of index 2 having as associated quadratic form the determinant function; hence the identity matrix will be an unit spacelike vector. While the determinant and Nomizu-Pinkall inner product differ only by a sign, we have chosen to work with the former as it seems more natural to us in terms of linear algebra; in any case, all results below can be easily reformulated in terms of $\langle\cdot, \cdot\rangle_{N P}$.

We start the second section with the general Hilbert-Schmidt inner product on $n \times n$ matrices and restrict gradually to $\operatorname{Sym}(2)$ performing also a comparison with the complementary $o(2)$. Several times we exemplify 
our computations with projectors and reflections. Our symmetric bilinear form is denoted $\langle\cdot, \cdot\rangle_{\text {det }}$ and is introduced and studied in the third section. We apply our construction to derive a rational symmetric function on the torus $T^{2}$ and consequently on the set of pairs of rays in the plane and we put our function in relationship with the case $n=2$ of a Morse function introduced and studied by Liviu Nicolaescu in [13] on arbitrary dimension $n \geq 2$. Also, we express our function with the help of well-known Hopf projection from $S^{3}$ to $S^{2}\left(\frac{1}{2}\right)$.

In the fourth section we present an approach based on complex numbers by using the Hopf invariant of any symmetric $2 \times 2$ matrix. With this formalism we compute both the critical points and the critical values of Morse function of Nicolaescu, again in the restricted dimension $n=2$. This complex approach identifies $\operatorname{Sym}(2)$ with the product $\mathbb{H}_{1}:=\mathbb{C} \times \mathbb{R}$ and the Heisenberg product of $\mathbb{H}_{1}$ is transported on $\operatorname{Sym}(2)$. We exemplify the Heisenberg product of two projectors and of two reflections and we obtain that the product of two projectors/reflections is not a projector/reflection.

In the last section we return to the Nomizu-Pinkall inner product and study new aspects concerning it, in addition to the direct relationship $\langle A, B\rangle_{N P}=-\langle A, B\rangle_{d e t}$. For example, the quadratic equation of critical points for a rational Morse function generates an almost contact structure on $\operatorname{Sym}(2)$ with the unitary matrix as associated Reeb vector. In fact, this structure is a cosymplectic one since its Reeb 1-form is exact.

\section{The Hilbert-Schmidt inner product}

Fix $n \in \mathbb{N}, n \geq 2$. The starting point of this note is the well-known Cartan decomposition of the Lie algebra $g l(n, \mathbb{R})=M_{n}(\mathbb{R})$ with respect to the negative transpose endomorphism $\cdot t$ :

$$
\left\{\begin{array}{l}
M_{n}(\mathbb{R})=\operatorname{Sym}(n) \oplus o(n)=\mathfrak{m} \oplus \mathfrak{h}, \quad \operatorname{dim}: n^{2}=\frac{n(n+1)}{2}+\frac{n(n-1)}{2} \\
{[\mathfrak{m}, \mathfrak{m}] \subset \mathfrak{h}, \quad[\mathfrak{m}, \mathfrak{h}] \subset \mathfrak{m}, \quad[\mathfrak{h}, \mathfrak{h}] \subset \mathfrak{h} .}
\end{array}\right.
$$

We remark here that the first works as well as the PhD Thesis of professor Aurel Bejancu are concerned with Banach-Lie groups; see, for example, [1] and [2]. Furthermore we fix $x \in \mathbb{R}^{n} \backslash\{\overline{0}\}$ which defines another direct sum decomposition:

$$
\mathbb{R}^{n}=\operatorname{span}\{x\} \oplus(\operatorname{span}\{x\})^{\perp}, \quad \operatorname{dim}: n=1+(n-1)
$$

which is an orthogonal decomposition with respect to the Euclidean inner product $\left\langle\cdot, \cdot>\right.$ of $\mathbb{R}^{n}$. Considering $x$ as being a column matrix i.e. as element in $M_{n, 1}(\mathbb{R})$ it follows the existence of two linear maps:

$$
\left\{\begin{array}{l}
\operatorname{sym}(x ; \cdot): \mathbb{R}^{n} \rightarrow \operatorname{Sym}(n), \quad \operatorname{sym}(x ; y):=\frac{1}{2\|x\|^{2}}\left(x \cdot y^{t}+y \cdot x^{t}\right) \\
o(x ; \cdot): \mathbb{R}^{n} \rightarrow o(n), \quad o(x ; y):=\frac{1}{2\|x\|^{2}}\left(x \cdot y^{t}-y \cdot x^{t}\right) .
\end{array}\right.
$$

Hence, every $y \in \mathbb{R}^{n}$ has the orthogonal decomposition $y=y_{x}^{\|}+y_{x}^{\perp}$ with:

$$
y_{x}^{\|}:=P_{x}(y)=\operatorname{sym}(x ; x)(y)=\frac{<x, y>}{\|x\|^{2}} x, \quad y_{x}^{\perp}=\left(I_{n}-\operatorname{sym}(x ; x)\right)(y)
$$

with $I_{n} \in \operatorname{Sym}(n)$ the identity matrix. It results the reflection with respect to $x$ :

$$
R_{x}: \mathbb{R}^{n} \rightarrow \operatorname{Sym}(n), \quad R_{x}:=2 P_{x}-I_{n}=2 \operatorname{sym}(x ; x)-I_{n} .
$$

The ambient space $M_{n}(\mathbb{R})$ of $(2.1)$ is Euclidean with respect to the Hilbert-Schmidt (or Frobenius) inner product:

$$
<A, B>_{H S}:=\frac{1}{n} \operatorname{Tr}\left(A \cdot B^{t}\right)=\frac{1}{n} \sum_{i, j=1}^{n} a_{j}^{i} b_{j}^{i}, \quad<A, I_{n}>_{H S}=\frac{1}{n} \operatorname{Tr}(A), \quad\left\|I_{n}\right\|_{H S}=1 .
$$

We point out that (2.1) is an orthogonal decomposition with respect to $<\cdot, \cdot\rangle_{H S}$. In the following we discuss the restriction of this inner product on both summands of (2.1) with a special view towards the case $n=2$.

\subsection{The restriction of $\langle\cdot, \cdot\rangle_{H S}$ on the second summand}

On the Lie algebra $\mathfrak{h}=o(n)$ we have:

$$
<A, B>_{o(n)}=-\frac{1}{n} \operatorname{Tr}(A \cdot B)
$$


which is an Ad-invariant inner product and thus it defines a bi-invariant Riemannian metric on the corresponding Lie group $O(n)=S O(n) \sqcup O^{-}(n)$ conform [12, p. 71]. We recall after [16, p. 11] that the manifold $\mathbb{E}_{n}:=G L(n, \mathbb{R}) / O(n)$ smoothly parametrizes the space of all (positive definite) inner products of $\mathbb{R}^{n}$; hence, the tangent space of $\mathbb{E}_{n}$ at the origin is identified with the space $\mathfrak{m}$. A natural Riemannian metric on $M$ is introduced in the exercise 6 from page 12 of the above book.

A straightforward computation gives:

$$
<o(x ; y), o(x ; z)>_{o(n)}=\frac{1}{2 n\|x\|^{4}}\left[\|x\|^{2}<y, z>-<x, y><x, z>\right]
$$

with the following consequences in which $\angle(x, y)$ denotes the angle between the vectors $x, y$ :

$$
\|o(x ; y)\|_{o(n)}=\frac{\|y\||\sin \angle(x, y)|}{\sqrt{2 n}\|x\|}, \quad y, z \in(\operatorname{span}\{x\})^{\perp} \rightarrow<o(x ; y), o(x ; z)>_{o(n)}=\frac{\langle y, z\rangle}{2 n\|x\|^{2}} .
$$

In particular, if $n=2$, then:

$$
A=A(a)=\left(\begin{array}{cc}
0 & -a \\
a & 0
\end{array}\right), B=A(b) \rightarrow<A, B>_{o(2)}=a b .
$$

The skew-symmetric endomorphism $J=A(1)$ is the (almost) complex structure of $\mathbb{R}^{2}$ and:

$$
<A, J>_{o(2)}=a=\|A(a)\|_{o(2)}, \quad\|J\|_{o(2)}=1 .
$$

The triple $\left.\left(\mathbb{R}^{2},<\cdot, \cdot\right\rangle, J\right)$ is a Kähler geometry i.e. $J$ is an isometry of the Euclidean plane and yields the symplectic 2 -form $\omega$ given by:

$$
\omega(\cdot, \cdot)=<\cdot, J \cdot>.
$$

Fix a regular plane curve $C: \bar{r}(t)=(x(t), y(t)) \in \mathbb{R}^{2}, t \in I \subseteq \mathbb{R}$. Its curvature function is:

$$
k(t)=\frac{x^{\prime}(t) y^{\prime \prime}(t)-y^{\prime}(t) x^{\prime \prime}(t)}{\left\|\bar{r}^{\prime}(t)\right\|^{3}}=\frac{\omega\left(\bar{r}^{\prime}(t), \bar{r}^{\prime \prime}(t)\right)}{\left\|\bar{r}^{\prime}(t)\right\|^{3}} .
$$

From the point of view of $G$-structures the geometry of $\left(\mathbb{R}^{2}, J\right)$ is controlled by the multiplicative group $G$ of CR-matrices:

$$
\mathbb{R}^{2}=\mathbb{C} \ni z:=x+i y \rightarrow C R(z):=\left(\begin{array}{cc}
x & -y \\
y & x
\end{array}\right)=x I_{2}+y J .
$$

The associated linear transformation of the plane is called similarity being a composition of a rotation and a homothety; we have:

$$
<C R\left(z_{1}\right), C R\left(z_{2}\right)>_{H S}=x_{1} x_{2}+y_{1} y_{2}=<z_{1}, z_{2}>_{\mathbb{R}^{2}} .
$$

The case $n=2$ of $(2.10)$ can be generalized as follows. To an arbitrary $A \in M_{n}(\mathbb{R})$ we associate:

$$
X_{A}=\left(\begin{array}{cc}
O_{n} & -A^{t} \\
A & O_{n}
\end{array}\right) \in o(2 n)
$$

and then:

$$
<X_{A}, X_{B}>_{o(2 n)}=\frac{1}{2 n} \operatorname{Tr}\left(A^{t} \cdot B+A \cdot B^{t}\right)=<A, B>_{H S(n)} .
$$

\subsection{The restriction of $\langle\cdot, \cdot\rangle_{H S}$ on the first summand}

On $\mathfrak{m}=\operatorname{Sym}(n)$ we have:

$$
<A, \tilde{A}>_{\operatorname{Sym}(n)}=\frac{1}{n} \operatorname{Tr}(A \cdot \tilde{A}), \quad<P_{x}, I_{n}>_{\operatorname{Sym}(n)}=\frac{1}{n}, \quad<R_{x}, I_{n}>_{\operatorname{Sym}(n)}=\frac{2}{n}-1 .
$$

We derive immediately:

$$
<\operatorname{sym}(x ; y), \operatorname{sym}(x, z)>_{\operatorname{Sym}(n)}=\frac{1}{2 n\|x\|^{4}}\left[\|x\|^{2}<y, z>+<x, y><x, z>\right]
$$


with the following consequences:

$$
\left\{\begin{array}{l}
\|\operatorname{sym}(x ; y)\|_{\operatorname{Sym}(n)}=\frac{\|y\| \sqrt{1+\cos ^{2} \angle(x, y)}}{\sqrt{2 n}\|x\|}, \\
y, z \in(\operatorname{span}\{x\})^{\perp} \mapsto<\operatorname{sym}(x ; y), \operatorname{sym}(x ; z)>_{\operatorname{Sym}(n)}=\frac{<y, z>}{2 n\|x\|^{2}}, \\
<P_{x}, P_{y}>_{\operatorname{Sym}(n)}=\frac{\cos ^{2} \angle(x, y)}{n} \in\left[0, \frac{1}{n}\right] \subseteq\left[0, \frac{1}{2}\right], \quad\left\|P_{x}\right\|_{\operatorname{Sym}(n)}=\frac{1}{\sqrt{n}}, \\
<R_{x}, R_{y}>_{\operatorname{Sym}(n)}=1-\frac{4 \sin ^{2} \angle(x, y)}{n} \in\left[1-\frac{4}{n}, 1\right], \quad\left\|R_{x}\right\|_{\operatorname{Sym}(n)}=1
\end{array}\right.
$$

and hence $P_{x} \perp_{\operatorname{Sym}(n)} P_{y}$ if and only if $x \perp y$ in $\mathbb{R}^{n}$. Also $R_{x} \perp_{S y m(n)} R_{y}$ if and only if $n=2$ and $x \perp y$.

For $n=2$ we have that $R_{x} \perp_{S y m(2)} I_{2},<P_{x}, P_{J x}>_{S y m(2)}=0$ and if $s$ is a natural parameter on the curve $C$ or $C$ is the circle $\mathcal{C}(O, R)$ with its trigonometrical parametrization $\bar{r}(s)=R(\cos s, \sin s)$ then:

$$
<P_{\bar{r}^{\prime}(s)}, P_{\bar{r}^{\prime \prime}(s)}>_{\operatorname{Sym}(2)}=0, \quad<R_{\bar{r}^{\prime}(s)}, R_{\bar{r}^{\prime \prime}(s)}>_{\operatorname{Sym}(2)}=-1 .
$$

If $A$ and $\tilde{A}$ from (2.18) are explicitly written then:

$$
A=\left(\begin{array}{ll}
a_{1}^{1} & a_{2}^{1} \\
a_{2}^{1} & a_{2}^{2}
\end{array}\right) \rightarrow<A, \tilde{A}>_{\operatorname{Sym}(2)}=\frac{1}{2}\left[a_{1}^{1} \tilde{a}_{1}^{1}+2 a_{2}^{1} \tilde{a}_{2}^{1}+a_{2}^{2} \tilde{a}_{2}^{2}\right] .
$$

Returning to the general case of $n$ for arbitrary $A \in g l(n, \mathbb{R})$ we have $A \cdot A^{t} \in \operatorname{Sym}(n)$ with:

$$
<A \cdot A^{t}, I_{n}>_{S y m(n)}=\|A\|_{H S}^{2} \geq 0 .
$$

In particular, if $A \in S L(n, \mathbb{R})$ then $A \cdot A^{t} \in \operatorname{Pos}_{\text {Sym }}^{1}(n)=$ the $\frac{(n-1)(n+2)}{2}$-dimensional manifold of all symmetric and positive definite matrices with determinant 1 . Moreover, the tangent space is well-known:

$$
T_{\left(A \cdot A^{t}\right)} \operatorname{Pos}_{\text {Sym }}^{1}(n)=\left\{B \in \operatorname{Sym}(n) ; \operatorname{Tr}\left(A^{-1} \cdot B \cdot\left(A^{t}\right)^{-1}\right)=0\right\} .
$$

\section{The determinant inner product on $\operatorname{Sym}(2)$}

In this section we restrict our setting to the 3-dimensional space $\operatorname{Sym}(2)$ since here the determinant function $\operatorname{det}: \operatorname{Sym}(2) \rightarrow \mathbb{R}$ is a quadratic form: $\operatorname{det} A=a_{1}^{1} a_{2}^{2}-\left(a_{2}^{1}\right)^{2}$; of course the determinant function is a quadratic form also on the ambient space $g l(2, \mathbb{R})$ but we restrict our setting in order to compare with the results of the previous section. Hence it corresponds in a polarization way to the symmetric bilinear form $\langle\cdot, \cdot\rangle_{\text {det }}$ : $\operatorname{Sym}(2) \times \operatorname{Sym}(2) \rightarrow \mathbb{R}$ given by:

$$
<A, \tilde{A}>_{\operatorname{det}}:=\frac{1}{2} \operatorname{Tr}\left(A \cdot \tilde{A}^{*}\right)=\frac{1}{2} \operatorname{Tr}\left(\tilde{A} \cdot A^{*}\right)=<A^{*}, \tilde{A}>_{\operatorname{Sym}(2)}, A^{*}=\left(\begin{array}{cc}
a_{2}^{2} & -a_{2}^{1} \\
-a_{2}^{1} & a_{1}^{1}
\end{array}\right) \in \operatorname{Sym}(2) .
$$

Its explicit form is:

$$
<A, \tilde{A}>_{\operatorname{det}}=\frac{1}{2}\left[a_{1}^{1} \tilde{a}_{2}^{2}-2 a_{2}^{1} \tilde{a}_{2}^{1}+a_{2}^{2} \tilde{a}_{1}^{1}\right]=\frac{1}{2} d(\operatorname{det})(A)(\tilde{A})=\frac{1}{2} d(\operatorname{det})(\tilde{A})(A), \quad\|A\|_{\operatorname{det}}^{2}=\operatorname{det} A,\left\|I_{2}\right\|_{\operatorname{det}}=1 .
$$

$\langle\cdot, \cdot\rangle_{\operatorname{det}}$ is a nondegenerate inner product on $\operatorname{Sym}(2)=\mathbb{R}^{3}$ with the null cone $N C$ provided by the cone $\operatorname{Sym}(2) \backslash G L(2, \mathbb{R})$ of singular matrices:

$$
N C \subset \mathbb{R}^{3}(x, y, z): x z=y^{2} .
$$

For example, each projector $P_{x}$ belongs to $N C$ and:

$$
\left\{\begin{array}{l}
<P_{x}, P_{y}>_{\operatorname{det}}=\frac{1}{2}\left[\omega\left(\frac{x}{\|x\|}, \frac{y}{\|y\|}\right)\right]^{2}=\frac{\cos ^{2} \angle(x, J y)}{2} \in\left[0, \frac{1}{2}\right] \\
<R_{x}, R_{y}>_{\text {det }}=\cos 2 \angle(x, J y) \in[-1,1], \quad \operatorname{det} R_{x}=-1=\left\|R_{x}\right\|_{\text {det }}^{2}
\end{array}\right.
$$

and hence each reflection $R_{x}$ is a timelike vector with respect to $\langle\cdot, \cdot\rangle_{\text {det }}$. We have $P_{x} \perp_{\text {det }} P_{y}$ if and only if $\angle(x, y) \in\{0, \pi\}$ and $R_{x} \perp_{\operatorname{det}} R_{y}$ if and only if $\angle(x, y) \in\left\{\frac{\pi}{4}, \frac{7 \pi}{4}\right\}$. 
For the considered curve $C$ we have:

$$
2<P_{\bar{r}^{\prime}(t)}, P_{\bar{r}^{\prime \prime}(t)}>_{\mathrm{det}}=\left[\frac{\left\|\bar{r}^{\prime}(t)\right\|^{2} k(t)}{\left\|\bar{r}^{\prime \prime}(t)\right\|}\right]^{2}
$$

and in particular, if $s$ is the natural parameter then:

$$
2<P_{\bar{r}^{\prime}(s)}, P_{\bar{r}^{\prime \prime}(s)}>_{\mathrm{det}}=-<R_{\bar{r}^{\prime}(s)}, R_{\bar{r}^{\prime \prime}(s)}>_{\mathrm{det}}=1 .
$$

If $\tilde{A}=\operatorname{diag}(\tilde{a}, \tilde{b})$ then:

$$
<A, \tilde{A}>_{\operatorname{det}}=\frac{1}{2}\left[a_{1}^{1} \tilde{b}+a_{2}^{2} \tilde{a}\right], \quad<A, I_{2}>_{\mathrm{det}}=\frac{1}{2} \operatorname{Tr}(A) .
$$

If one of the given matrices, say $\tilde{A}$, belongs to $\operatorname{Sym}(2) \cap G L(2, \mathbb{R})$ then:

$$
<A, \tilde{A}>_{\operatorname{det}}=\frac{\operatorname{det} \tilde{A}}{2} \operatorname{Tr}\left(A \cdot \tilde{A}^{-1}\right) .
$$

In particular, if $\tilde{A} \in O^{-}(2) \subset \operatorname{Sym}(2) \cap G L(2, \mathbb{R})$ then:

$$
<A, \tilde{A}>_{\mathrm{det}}=-\frac{1}{2} \operatorname{Tr}\left(A \cdot \tilde{A}^{t}\right)=-\frac{1}{2} \operatorname{Tr}(A \cdot \tilde{A})=-<A, \tilde{A}>_{\operatorname{Sym}(2)}
$$

and we remark a formal similarity with the relation (2.7) for $n=2$. We point out here a remarkable application of the relation (3.8). Fix $S \subset \mathbb{R}^{3}$ a regular surface and denote, as usually, $I$ and $I I$ its two fundamental forms. Then, its mean curvature is:

$$
H=\frac{1}{\operatorname{det} I}<I, I I>_{\operatorname{det}}
$$

and hence $S$ is a minimal surface if and only if $I \perp_{\text {det }} I I$ !

We present now an application of the $O^{-}(2)$ case. Let $T^{2}:=S^{1} \times S^{1}$ be the algebraic 2-torus and its points $(z, \tilde{z}) \in T^{2}$ expressed in the exponential form: $z=e^{i u}, \tilde{z}=e^{i \tilde{u}}$. Remark that if instead of the pair $\left(z_{1}, z_{2}\right)$ we work with the matrix $\operatorname{diag}\left(z_{1}, z_{2}\right)$ then we identify $T^{2}$ with the maximal torus of $U(2)$. Recall the correspondence of $S^{1} \simeq S O(2)$ with $O^{-}(2)$ (which is not a group, but in relationship with the axial symmetries of the plane conform [9]):

$$
S^{1} \ni z=e^{i u} \rightarrow R_{e^{i \frac{u}{2}}}=\left(\begin{array}{cc}
\cos u & \sin u \\
\sin u & -\cos u
\end{array}\right) \in O^{-}(2) \cap \operatorname{sl}(2, \mathbb{R}) .
$$

Hence, we define a map $f_{\text {det }}: T^{2} \rightarrow \mathbb{R}$ by:

$$
f_{\operatorname{det}}(z, \tilde{z})=<R_{e^{i \frac{u}{2}}}, R_{e^{i \frac{\tilde{u}}{2}}}>_{\operatorname{det}}=-<R_{e^{i \frac{u}{2}}}, R_{e^{i \frac{\tilde{u}}{2}}}>_{S y m(2)} .
$$

Its properties are immediately:

i) is symmetric: $f_{\operatorname{det}}(z, \tilde{z})=f_{\operatorname{det}}(\tilde{z}, z)$,

ii) its relationship with the determinant function: $f_{\operatorname{det}}(z, z)=-1=\operatorname{det} R_{e^{i \frac{u}{2}}}=-\left\|R_{e^{i \frac{u}{2}}}\right\|_{S y m(2)}^{2}$,

iii) its explicit expression is:

$$
f_{\operatorname{det}}(z, \tilde{z})=-\cos (u-\tilde{u})=\frac{1}{2}\|z-\tilde{z}\|_{\mathbb{R}^{2}}^{2}-1=\frac{1}{2} d_{\mathbb{R}^{2}}^{2}(z, \tilde{z})-1=-\cos d_{S^{1}}(z, \tilde{z})
$$

with $d_{\mathbb{R}^{2}}$ and $d_{S^{1}}$ the Euclidean distance and the spherical $\left(S^{1}\right)$ distance respectively, iv) the relationship with the product structure of $S^{1}$ :

$$
f_{\text {det }}\left(z_{1} \cdot z_{2}, z_{3}\right)=f_{\text {det }}\left(z_{2}, z_{1}^{-1} \cdot z_{3}\right)
$$

is a direct consequence of (3.12).

A geometrical interpretation of $f_{\text {det }}$ can be given by the Euclidean geometry of the plane $\mathbb{R}^{2}(x, y)$. For any $t \in \mathbb{R}$ let $r_{t} \in \mathbb{R}^{2}$ be the ray with slope $t$ given by $r_{t}: y=t x$ with $x \geq 0$ and let us consider $t=\tan \frac{u}{2}$. Hence $r_{t}$ is "half" of the line $L_{t}=r_{t} \sqcup r_{-t}$ and its is well-known that $L_{t}$ is characterized by its orthogonal projector $P_{L_{t}}$ which is exactly $R_{e^{i} \frac{u}{2}}$. It follows a symmetrical function on the set of pairs of rays:

$$
f_{\text {det }}\left(r_{t}, r_{\tilde{t}}\right)=-\cos 2(\arctan t-\arctan \tilde{t}), \quad f_{\text {det }}\left(r_{t}, r_{t}\right)=-1
$$


with the explicit rational expression:

$$
f_{\operatorname{det}}\left(r_{t}, r_{\tilde{t}}\right)=\frac{t^{2}+\tilde{t}^{2}-1-4 t \tilde{t}-(t \tilde{t})^{2}}{\left(1+t^{2}\right)\left(1+\tilde{t}^{2}\right)}=\frac{(t-\tilde{t}+1+t \tilde{t})(t-\tilde{t}-1-t \tilde{t})}{\left(1+t^{2}\right)\left(1+\tilde{t}^{2}\right)} .
$$

By adapting the chordal distance $\chi$ of the Riemann sphere ([10, p. 63]) to the unit circle we have:

$$
\chi(t, \tilde{t}):=\frac{|t-\tilde{t}|}{\sqrt{1+t^{2}} \sqrt{1+\tilde{t}^{2}}} \leq 1
$$

and then:

$$
f_{\operatorname{det}}\left(r_{t}, r_{\tilde{t}}\right)=\chi^{2}(t, \tilde{t})-\left[\frac{1+t \tilde{t}}{\sqrt{1+t^{2}} \sqrt{1+\tilde{t}^{2}}}\right]^{2} \leq 1-\left[\frac{1+t \tilde{t}}{\sqrt{1+t^{2}} \sqrt{1+\tilde{t}^{2}}}\right]^{2} .
$$

For the example of positive axis $O x^{+}=r_{0}$ i.e. $u=0$ and the first bisectrix $B_{1}^{+}: y=x \geq 0$ i.e. $\tilde{u}=\frac{\pi}{2}$ we have: $f_{\text {det }}\left(O x^{+}, B_{1}^{+}\right)=0$. The pair of orthogonal rays $\left(r_{t}, r_{-\frac{1}{t}}\right)$ corresponds to $\tilde{u}=u+\pi$ and then: $f_{\text {det }}\left(r_{t}, r_{-\frac{1}{t}}\right)=1$. Also, for the pair of opposite rays $\left(r_{t}, r_{-t}\right)$ i.e. $\tilde{u}=-u$ we have:

$$
f_{\operatorname{det}}\left(r_{t}, r_{-t}\right)=-\cos (2 u), \quad u=2 \arctan t, \quad \chi(\tan u, \tan \tilde{u})=|\sin (u-\tilde{u})| .
$$

We remark that in the paper [13], by using the matrix $R_{e^{i \cdot 0}}=\operatorname{diag}(1,-1)$, thus corresponding to the line $O x=L_{0}$, the function:

$$
f_{R_{0}}\left(L_{t}\right)=-2 \cos u=2 f_{\text {det }}\left(r_{t}, O x^{+}\right)
$$

is obtained as a Morse function on the Grassmannian $G_{1}(2) \simeq S^{1} \simeq S O(2) / S O(1)$ of lines in $\mathbb{R}^{2}$. Hence, our flow $t \in \mathbb{R} \rightarrow f_{\text {det }}\left(r_{t}, O x^{+}\right)$is "half" of the Morse flow of Nicolaescu as can be expected from our choice of considering rays instead of lines.

We finish this section with an interpretation of the formula (3.12) in terms of Hopf bundle $H: S^{3} \subset \mathbb{C}^{2} \rightarrow S^{2}\left(\frac{1}{2}\right) \subset \mathbb{R} \times \mathbb{C}:$

$$
H(z, w)=\left(\frac{1}{2}\left(|z|^{2}-|w|^{2}\right), z \bar{w}\right) .
$$

Since the Clifford torus $\frac{1}{\sqrt{2}} T^{2} \subset S^{3}$ one follows:

$$
\pi_{2} \circ H\left(\frac{1}{\sqrt{2}}(z, \tilde{z})\right)=\frac{1}{2} e^{i(u-\tilde{u})}
$$

and we have the claimed interpretation:

$$
\frac{1}{\sqrt{2}} f_{\text {det }}: \frac{1}{\sqrt{2}} T^{2} \subset S^{3} \rightarrow \mathbb{R}, \quad \frac{1}{\sqrt{2}} f_{\text {det }}=-2 \operatorname{Re}\left(\pi_{2} \circ H\right) .
$$

Remark that in terms of complex numbers $z:=x+i y$ the line $L_{t}$ has the equations:

$$
L_{t}:(1-t i) \cdot z \in \mathbb{R} \leftrightarrow L_{t}: \operatorname{Im}((1-t i) \cdot z)=0 .
$$

More generally, let $z_{0}, v \in \mathbb{C}$ with $v \neq 0$. The line $L_{z_{0}, v}: z(\lambda)=z_{0}+\lambda v \in \mathbb{C}$ with the real parameter $\lambda$ has the equation:

$$
L_{z_{0}, v}: \operatorname{Im}\left(\left(z-z_{0}\right) \cdot \bar{v}\right)=0 .
$$

\section{A complex approach to $<\cdot, \cdot\rangle_{\text {det }}$ and the Heisenberg product on $\operatorname{Sym}(2)$}

Let $H(2) \subset M_{2}(\mathbb{C})$ be the 4-dimensional real space of Hermitian $2 \times 2$ matrices with its Pauli basis:

$$
\sigma_{0}=I_{2}, \quad \sigma_{1}=\left(\begin{array}{cc}
0 & 1 \\
1 & 0
\end{array}\right)=R_{e^{i \frac{\pi}{4}}}, \quad \sigma_{2}=\left(\begin{array}{cc}
0 & -i \\
i & 0
\end{array}\right)=i J, \quad \sigma_{3}=\operatorname{diag}(1,-1)=R_{e^{i \cdot 0}} .
$$


Hence $J=\sigma_{1} \sigma_{3}=(-i) \sigma_{2}$ and $\operatorname{Sym}(2)=H(2) \cap\left\{\right.$ hyperplane $\left.: \sigma_{2}^{*}=0\right\}$ with the basis $\left\{\sigma_{0}, \sigma_{1}, \sigma_{3}\right\}$ orthonormal with respect to $\left\langle\cdot, \cdot>_{S y m(2)}\right.$ and $\left\{\sigma_{i}^{*} ; 0 \leq i \leq 4\right\}$ the dual basis in $H(2)^{*}$. This basis is only orthogonal with respect to $\langle\cdot, \cdot\rangle_{\text {det }}$ with $\left\|\sigma_{0}\right\|_{\text {det }}^{2}=1=-\left\|\sigma_{1}\right\|_{\text {det }}^{2}=-\left\|\sigma_{3}\right\|_{\text {det }}^{2}$ which means that the index of the symmetric bilinear form $\langle\cdot, \cdot\rangle_{\text {det }}$ is 2 with $\sigma_{0}$ as spacelike vector and $\sigma_{1}$ and $\sigma_{3}$ as timelike vectors. We note that a trace-type inner product of complex $2 \times 2$ matrices appears in the formula (3.20) of [7].

In [5] (see also [6, p. 89]) an embedding of $\operatorname{Sym}(2)$ into $H(2)$ is introduced by the map:

$$
\left\{\begin{array}{l}
\operatorname{Sym}(2) \ni \Gamma=\Gamma(a, b, c):=\left(\begin{array}{cc}
a & b \\
b & c
\end{array}\right) \mapsto \Gamma^{c}:=\left(\begin{array}{cc}
B & \bar{A} \\
A & B
\end{array}\right) \in H(2) \cap\left\{\text { hyperplane }: \sigma_{3}^{*}=0\right\}, \\
2 B:=\operatorname{Tr} \Gamma=a+c \in \mathbb{R}, A:=\frac{a-c}{2}-b i \in \mathbb{C} .
\end{array}\right.
$$

In the book [11, p. 56] the complex number $A$ is denoted $L(\Gamma)$ and is called the Hopf invariant of $\Gamma$. The initial matrix $\Gamma$ can be recovered by its image $\Gamma^{c}$ through the relations:

$$
a=B+\operatorname{Re}(A), \quad c=B-\operatorname{Re}(A), \quad b=-\operatorname{Im}(A)
$$

and then the following decompositions hold:

$$
\Gamma=B \sigma_{0}+(-\operatorname{Im}(A)) \sigma_{1}+(\operatorname{Re}(A)) \sigma_{3}, \quad \Gamma^{c}=B \sigma_{0}+(\operatorname{Re}(A)) \sigma_{1}+(\operatorname{Im}(A)) \sigma_{2} .
$$

For the example of projectors and reflections we have:

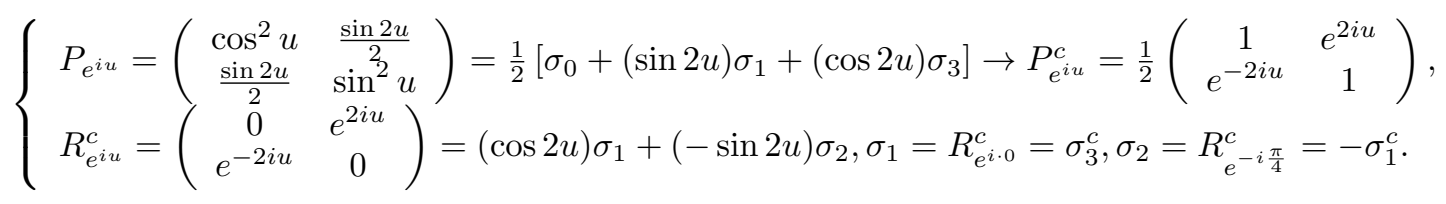

The map ${ }^{c}$ preserves $I_{2}=\sigma_{0}$ and the inner product $\langle\cdot, \cdot\rangle_{S y m(2)}$ corresponds to the restriction of the HilbertSchimdt inner product of $H(2)$ to its hyperplane $\sigma_{2}^{*}=0$ :

$$
\left\{\begin{array}{l}
I_{2}^{c}=I_{2}, \quad<\Gamma_{1}, \Gamma_{2}>_{\operatorname{Sym}(2)}=\frac{1}{2} \operatorname{Tr}\left(\Gamma_{1}^{c} \cdot \Gamma_{2}^{c}\right)=B_{1} B_{2}+\operatorname{Re}\left(A_{1} \bar{A}_{2}\right) \\
\|\Gamma\|_{\text {Sym }(2)}^{2}=B^{2}+|A|^{2}=\left(\|B\|_{\mathbb{R}^{1}}\right)^{2}+\left(\|A\|_{\mathbb{R}^{2}}\right)^{2}
\end{array}\right.
$$

Moreover, the map.$^{c}$ preserves the algebraic invariants:

$$
\operatorname{Tr} \Gamma=\operatorname{Tr} \Gamma^{c}=2 B, \quad \operatorname{det} \Gamma=\operatorname{det} \Gamma^{c}=B^{2}-|A|^{2}
$$

which implies immediately that the equation of the null cone is $N C: B=|A| \geq 0$ and:

$$
\left\{\begin{array}{l}
<\Gamma_{1}, \Gamma_{2}>_{\text {det }}=B_{1} B_{2}-\left(\operatorname{Re} A_{1} \cdot \operatorname{Re} A_{2}+\operatorname{Im} A_{1} \cdot \operatorname{Im} A_{2}\right)=<B_{1}, B_{2}>_{\mathbb{R}^{1}}-<A_{1}, A_{2}>_{\mathbb{R}^{2}}, \\
\|\Gamma\|_{\operatorname{det}}^{2}=B^{2}-|A|^{2}=\left(\|B\|_{\mathbb{R}^{1}}\right)^{2}-\left(\|A\|_{\mathbb{R}^{2}}\right)^{2} .
\end{array}\right.
$$

The first formula (4.8) appears in Remark 3.1 of [8]. The matrix $\Gamma$ is positive-definite i.e. its eigenvalues $\lambda_{1,2}$ are positive if and only if $a=B+\operatorname{Re} A>0$ and $B^{2}>|A|^{2}$. The general formula of eigenvalues is: $\lambda_{1,2}(\Gamma)=B \pm|A|$.

In fact, the map $\cdot^{c}$ can be considered as the restriction to $\operatorname{Sym}(2)=\mathbb{R}^{3}$ of the linear endomorphism $T^{c}: \mathbb{R}^{4} \rightarrow$ $\mathbb{R}^{4}$ :

$$
T^{c}=\left(\begin{array}{cccc}
1 & 0 & 0 & 0 \\
0 & 0 & 0 & 1 \\
0 & -1 & 0 & 0 \\
0 & 0 & -1 & 0
\end{array}\right) \in M_{4}(\mathbb{R}), \quad T^{c}(x, y, z, t)=(x, t,-y,-z) .
$$

This endomorphism belongs to the Lie group $S O(4)=\mathbb{R}^{6}$ having $\operatorname{Tr} T^{c}=1=\operatorname{det} T^{c}$ and the eigevalues $\lambda_{1}=$ $\lambda_{2}=1, \lambda_{3}=\overline{\lambda_{4}}=e^{\frac{2 \pi i}{3}}$. It results that $T^{c}$ is a third-order root of the unit matrix: $\left(T^{c}\right)^{3}=I_{4}$. It follows a second third-order root of the unit matrix:

$$
T_{r}^{c}=\left(\begin{array}{ccc}
0 & 0 & 1 \\
-1 & 0 & 0 \\
0 & -1 & 0
\end{array}\right) \in S O(3), \quad\left(T_{r}^{c}\right)^{3}=I_{3}, \quad\left(T_{r}^{c}\right)(x, y, z)=(z,-x,-y) .
$$


In the following we study in this setting the previous called function of Nicolaescu from [13]. For a fixed $\Gamma \in \operatorname{Sym}(2)$ the function is of height type:

$$
f_{\Gamma}: G_{1}(2) \subset \operatorname{Sym}(2) \rightarrow \mathbb{R}, \quad f_{\Gamma}\left(L_{t}\right)=f_{\Gamma}\left(P_{L_{t}}\right)=f_{\Gamma}(t):=<\Gamma, P_{L_{t}}>_{S y m(2)} .
$$

With $\Gamma=\Gamma(a, b, c)$ as in (4.2) it follows a rational expression of $f_{\Gamma}$ :

$$
f_{\Gamma}(t)=\frac{a+2 b t+c t^{2}}{1+t^{2}}, \quad t=\tan \frac{\varphi}{2} \rightarrow f_{\Gamma}(\varphi)=B+<A, e^{-i \varphi}>_{\mathbb{R}^{2}}
$$

and its critical points are solutions to:

$$
b t^{2}+(a-c) t-b=0=(b+c t)-t(a+b t) .
$$

It is amazing that the same equation (4.11) gives the fixed points of the linear fractional transformation:

$$
F_{\Gamma}: S^{1} \simeq \mathbb{R} \cup\{\infty\} \rightarrow S^{1}, \quad F_{\Gamma}(t):=\frac{c t+b}{b t+a}=t^{\prime}
$$

which, in an old fashion style, can be written as: $b t t^{\prime}-c t+a t^{\prime}-b=0$. The second equality says that the critical $L_{t}$ are orthogonal invariant lines through the linear action of $\Gamma$ on $\mathbb{R}^{2}$. Supposing that $b=-\operatorname{Im}(A) \neq 0$ the discriminant of the second-order equation (4.11) is:

$$
\Delta=(a-c)^{2}+4 b^{2}=4|A|^{2}>0
$$

and thus the critical points of $f_{\Gamma}$ are:

$$
t_{ \pm}=\frac{\operatorname{Re}(A) \mp|A|}{\operatorname{Im}(A)} .
$$

Expressing $A$ exponentially as $A=|A| e^{i u}$ with $u=u(\Gamma)$ it results:

$$
\left\{\begin{array}{l}
t_{+}=-\tan \frac{u}{2}, \quad t_{-}=\cot \frac{u}{2}, \\
f_{\Gamma}\left(t_{+}\right)=a \cos ^{2} \frac{u}{2}-b \sin u+c \sin ^{2} \frac{u}{2}=B+|A|=\lambda_{1}(\Gamma), \\
f_{\Gamma}\left(t_{-}\right)=a \sin ^{2} \frac{u}{2}+b \sin u+c \cos ^{2} \frac{u}{2}=B-|A|=\lambda_{2}(\Gamma) .
\end{array}\right.
$$

If $b=0$ i.e. $\Gamma$ is a diagonal matrix then $f_{\Gamma}$ has $t=0$ as unique critical point and the illustrative example of the paper [3, p. 258] is of this type with $a=1$ and $c \in(0,1)$. The Hessian of the general $f_{\Gamma}$ is:

$$
\frac{1}{2} f_{\Gamma}^{\prime \prime}(t)=\frac{(c-a)-6 b t-3(c-a) t^{2}+2 b t^{3}}{\left(1+t^{2}\right)^{3}} .
$$

Particular cases and examples I) It is well-known that the chart map $\mathbb{R} \ni t \mapsto(\cos u, \sin u) \in S^{1}$ for $u=$ $2 \arctan t$ is the stereographic projection of $S^{1}$ from its point $P(-1,0)$ and is useful to derive the general expression of Pythagorean triples. We have then the trigonometric parametrization of $S^{1}$ :

$$
x(t)=\frac{1-t^{2}}{1+t^{2}}, \quad y(t)=\frac{2 t}{1+t^{2}}, \quad P=\lim _{t \rightarrow \infty}(x(t), y(t)) \rightarrow S^{1}: z(t)=\frac{1+t i}{1-t i}, \quad t \in \mathbb{R} \cup\{\infty\} .
$$

Hence the scalar combination $a \cdot x(t)+b \cdot y(t)$ has the expression (4.10) with $c=-a$ which means a traceless matrix $\Gamma \in \operatorname{Sym}(2)$.

We point out that for $\Gamma \in \operatorname{Sym}(2)$ with $b \neq 0$ the following statements are equivalent: i) $\Gamma$ is traceless; ii) $B=0$; iii) the linear fractional transformation $F_{\Gamma}$ is an involution: $F_{\Gamma} \circ F_{\Gamma}=1_{S^{1}}$. This is the case of:

a) the inversion $R(t)=\frac{1}{t}$ with:

$$
R=\left(\begin{array}{ll}
0 & 1 \\
1 & 0
\end{array}\right)=R_{e^{i \frac{\pi}{4}}} \in O^{-}(2) \cap \operatorname{sl}(2, \mathbb{R}), \quad A(R)=-i \in \mathbb{C} \backslash \mathbb{R}
$$

b) the map $F_{\Gamma}(t)=\frac{1-t}{1+t}$ corresponding to:

$$
\Gamma=\left(\begin{array}{cc}
1 & 1 \\
1 & -1
\end{array}\right), \quad A(\Gamma)=A(R)=-i .
$$


Hence $R^{c}=\Gamma^{c}=-\sigma_{2}$. The fixed (or double) points of $R$ are \pm 1 while that of $F_{\Gamma}$ are $t_{1,2}=-1 \pm \sqrt{2}$.

II) Fix three distinct points $t_{1}, t_{2}, t_{3}$. Through the cross-ratio formula we define the linear fractional:

$$
F_{\Gamma}(t):=\left(t_{1}, t_{2} ; t_{3}, t\right)=\frac{t-t_{2}}{t-t_{1}}: \frac{t_{3}-t_{2}}{t_{3}-t_{1}} .
$$

We identify the coefficients: $c=t_{3}-t_{1} \neq 0, b=t_{3}-t_{2}(\neq 0)=t_{2}\left(t_{1}-t_{3}\right), a=-t_{1} b$ and the equality from the expression of $b$ reads as:

$$
t_{3}=\frac{t_{2}\left(1+t_{1}\right)}{1+t_{2}}
$$

This expression is different to $t_{1}$ and $t_{2}$ for $t_{2} \neq 0$ and hence we impose $t_{2} \notin\{0,-1\}$ and different to $t_{1}$. The final expression of the coefficients is:

$$
\left\{\begin{array}{l}
a=\frac{t_{1} t_{2}\left(t_{2}-t_{1}\right)}{1+t_{2}}, \quad b=\frac{t_{2}\left(t_{1}-t_{2}\right)}{1+t_{2}}, \quad c=\frac{t_{2}-t_{1}}{1+t_{1}}, \\
A(\Gamma)=\frac{t_{2}-t_{1}}{1+t_{2}}\left(\frac{t_{1} t_{2}-1}{2}+i t_{2}\right), \quad B(\Gamma)=\frac{\left(t_{2}-t_{1}\right)\left(t_{1} t_{2}+1\right)}{2}, \quad \operatorname{det} \Gamma=-\frac{t_{2}\left(t_{2}-t_{1}\right)^{3}}{\left(1+t_{2}\right)^{2}} \neq 0 .
\end{array}\right.
$$

The discriminant $\Delta$ of $(4.12)$ is:

$$
\Delta=\left(\frac{t_{2}-t_{1}}{1+t_{2}}\right)^{2}\left[\left(t_{1} t_{2}-1\right)^{2}+4 t_{2}^{2}\right]
$$

Returning to the map $\cdot^{c}$ we remark that it identifies $\operatorname{Sym}(2)$ with the product $\mathbb{C} \times \mathbb{R}$. Recall that this set when is endowed with the product:

$$
\left(z_{1}, t_{1}\right) *\left(z_{2}, t_{2}\right):=\left(z_{1}+z_{2}, t_{1}+t_{2}+\operatorname{Im}\left(\overline{z_{1}} z_{2}\right)\right)=\left(z_{1}+z_{2}, t_{1}+t_{2}-\omega\left(z_{1}, z_{2}\right)\right)
$$

becomes a group, called Heisenberg and denoted $\mathbb{H}_{1}$. It follows a Heisenberg group law on $\operatorname{Sym}(2)$ :

$$
\Gamma_{1} * \Gamma_{2}:=\Gamma\left((a, b, c):=\left(A_{1}, B_{1}\right) *\left(A_{2}, B_{2}\right)\right)
$$

or, explicitly:

$$
a:=a_{1}+a_{2}+\frac{b_{1}}{2}\left(a_{2}-c_{2}\right)-\frac{b_{2}}{2}\left(a_{1}-c_{1}\right), \quad c:=c_{1}+c_{2}+\frac{b_{1}}{2}\left(a_{2}-c_{2}\right)-\frac{b_{2}}{2}\left(a_{1}-c_{1}\right), \quad b=b_{1}+b_{2} .
$$

We derive immediately that $O_{2}=\Gamma(0,0,0)$ is the neutral element, the inverse is $[\Gamma(a, b, c)]^{-1}=\Gamma(-a,-b,-c)$ while the squares are $\Gamma * \Gamma=2 \Gamma$ and $\Gamma * I_{2}=\Gamma+I_{2}$. For the example of projectors and reflections we have:

$$
\begin{gathered}
P_{e^{i u_{1}}} * P_{e^{i u_{2}}}: a=\cos ^{2} u_{1}+\cos ^{2} u_{2}+\frac{1}{4} \sin 2\left(u_{1}-u_{2}\right), \quad c=\sin ^{2} u_{1}+\sin ^{2} u_{2}+\frac{1}{4} \sin 2\left(u_{1}-u_{2}\right), \\
b=\frac{1}{2}\left(\sin 2 u_{1}+\sin 2 u_{2}\right), \\
R_{e^{i u_{1}}} * R_{e^{i u_{2}}}: a=\cos 2 u_{1}+\cos 2 u_{2}+\sin 2\left(u_{1}-u_{2}\right), \quad c=-\cos 2 u_{1}-\cos 2 u_{2}+\sin 2\left(u_{1}-u_{2}\right), \\
b=\sin 2 u_{1}+\sin 2 u_{2} .
\end{gathered}
$$

An explicit example is:

$$
\sigma_{1} * \sigma_{3}=\left(\begin{array}{cc}
2 & 1 \\
1 & 0
\end{array}\right)
$$

and a direct argument proves that the product of two projectors is not a projector; indeed the trace of a projector is 1 while the corresponding sum for (4.18) is: $a+c=2+\frac{1}{2} \sin 2\left(u_{1}-u_{2}\right) \geq 2-\frac{1}{2}=\frac{3}{2}$. Analogous a product of reflections is not a reflection; since a reflection belongs to $s l(2, \mathbb{R})$ the corresponding sum $a+c$ for (4.19) implies $2\left(u_{2}-u_{2}\right) \in \mathbb{Z} \pi$ and then the corresponding product of reflections is $R * R=2 R$ or $O_{2}$ and both these matrices are not reflections.

We finish this section with the well-known remark that $\mathbb{H}_{1}$ can be though as a subgroup in $U(1,2)$ by considering:

$$
\Lambda(z, t):=\left(\begin{array}{ccc}
1+\frac{|z|^{2}}{2}+i t & \bar{z} & -\frac{|z|^{2}}{2}-i t \\
z & 1 & z \\
\frac{|z|^{2}}{2}+i t & \bar{z} & 1-\frac{|z|^{2}}{2}-i t
\end{array}\right), \quad \operatorname{Tr} \Lambda(z, t)=3, \quad \operatorname{det} \Lambda(z, t)=1-2|z|^{2} .
$$




\section{Back to the Nomizu-Pinkall inner product}

In this last section we return to the inner product $\langle\cdot, \cdot\rangle_{N P}$ and its explicit formula:

$$
<A, B>_{N P}=\frac{1}{2}\left[a_{2}^{1} b_{1}^{2}+a_{1}^{2} b_{2}^{1}-a_{1}^{1} b_{2}^{2}-a_{2}^{2} b_{1}^{1}\right] .
$$

We recall the relation $\langle A, A\rangle_{N P}=-\operatorname{det} A$. We obtain also:

$$
<P_{e^{i u_{1}}}, R_{e^{i u_{2}}}>_{N P}=<P_{e^{i u_{1}}}, R_{e^{i u_{2}}}>_{S y m(2)}=\frac{1}{2} \cos 2\left(u_{1}-u_{2}\right)
$$

and then $P_{e^{i u_{1}}} \perp_{\text {Sym }(2)} R_{e^{i u_{2}}}$ if and only if $4\left(u_{1}-u_{2}\right) \in(2 \mathbb{Z}+1) \frac{\pi}{2}$. The orthonormal basis used in [14, p. 208] is $\left\{E_{1}=I_{2}, E_{2}=\sigma_{3}, E_{3}=\sigma_{1}, E_{4}=J\right\}$ with:

$$
\left\|E_{1}\right\|_{N P}^{2}=\left\|E_{4}\right\|_{N P}^{2}=-1, \quad\left\|E_{2}\right\|_{N P}^{2}=\left\|E_{3}\right\|_{N P}^{2}=+1 .
$$

It results that $\operatorname{Sym}(2)$ is the hyperplane $\operatorname{span}\left\{E_{1}, E_{2}, E_{3}\right\}$ of $\operatorname{gl}(2, \mathbb{R})$ and the decomposition of arbitrary $\Gamma \in \operatorname{Sym}(2)$ is:

$$
\Gamma=\Gamma\left(a_{1}, a_{2}, a_{3}\right):=a_{1} E_{1}+a_{2} E_{2}+a_{3} E_{3}=\left(\begin{array}{cc}
a_{1}+a_{2} & a_{3} \\
a_{3} & a_{1}-a_{2}
\end{array}\right)
$$

which in our complex formalism means: $B=a_{1}$ and $A=a_{2}-a_{3} i$. The hyperplane $\operatorname{sl}(2, \mathbb{R})$ of $g l(2, \mathbb{R})$ is $\operatorname{span}\left\{E_{2}, E_{3}, E_{4}\right\}$.

Another important class of symmetric $2 \times 2$ matrices, useful in 2D dynamics of Teichmüller flows ([17, p. 95]), are $\operatorname{Exp}(t)=\operatorname{diag}\left(e^{t}, e^{-t}\right) \in S L(2, \mathbb{R})$ for any $t \in \mathbb{R}$. It follows:

$$
<\operatorname{Exp}\left(t_{1}\right), \operatorname{Exp}\left(t_{2}\right)>_{S y m(2)}=\cosh \left(t_{1}+t_{2}\right) \geq 1, \quad<\operatorname{Exp}\left(t_{1}\right), \operatorname{Exp}\left(t_{2}\right)>_{\text {det }}=\cosh \left(t_{1}-t_{2}\right) \geq 1 .
$$

Hence in this class of matrices there are no orthogonal pairs both to $\langle\cdot, \cdot\rangle_{\text {Sym }(2)}$ and $\langle\cdot, \cdot\rangle_{\text {det }}$.

Another method to obtain new symmetric matrices is provided by the function $f_{\Gamma}$ of (4.10) and the equation (4.11) of its critical points. More precisely, we define the function $\operatorname{Cr}: \operatorname{Sym}(2) \rightarrow \operatorname{Sym}(2) \cap \operatorname{sl}(2, \mathbb{R})$ :

$$
C r(\Gamma(a, b, c)):=\Gamma\left(b, \frac{c-a}{2},-b\right)=\left(\begin{array}{cc}
b & \frac{c-a}{2} \\
\frac{c-a}{2} & -b
\end{array}\right), \quad A(C r(\Gamma))=i A(\Gamma), \quad B(C r(\Gamma))=0 .
$$

Its unique fixed point is the null matrix $\mathrm{O}_{2}$ and in terms of Pauli basis we have:

$$
\operatorname{Cr}(\Gamma)=\frac{c-a}{2} \sigma_{1}+b \sigma_{3}=(-\operatorname{Re}(A)) \sigma_{1}+(-\operatorname{Im}(A)) \sigma_{3}
$$

which means that the linear endomrphism $C r$ of $\operatorname{Sym}(2)$ has the rank two. Its kernel is $\mathbb{R} \cdot I_{2}$ and as example $\operatorname{Cr}\left(P_{e^{i u}}\right)=R_{e^{i\left(\frac{3 \pi}{2}+u\right)}} ;$ also from $\operatorname{det} C r(\Gamma)=\operatorname{det} \Gamma-\frac{(a+c)^{2}}{4}$ it results that $\operatorname{det} C r(\Gamma) \leq \min \{0, \operatorname{det} \Gamma\}$. An important conclusion is that $\operatorname{Cr}(\Gamma)$ is orthogonal to $\Gamma$ with respect to both inner products:

$$
<\Gamma, \operatorname{Cr}(\Gamma)>_{\operatorname{Sym}(2)}=0=<\Gamma, \operatorname{Cr}(\Gamma)>_{\text {det }} .
$$

With the matrix representation of the endomorphism $C r$ :

$$
C r=\left(\begin{array}{ccc}
0 & 1 & 0 \\
-\frac{1}{2} & 0 & \frac{1}{2} \\
0 & -1 & 0
\end{array}\right)
$$

we deduce that $C r$ is an $f$-structure ([15]) i.e. it satisfies:

$$
C r^{3}+C r=0
$$

but having the maximally (even) rank is actually an almost contact structure on $\operatorname{Sym}(2)$. Its Reeb vector $\xi$ is the unique vector of the basis of its kernel hence: $\xi=\sigma_{0}=\Gamma(1,0,1)$. From the first decomposition (4.4) it results that the dual of the Reeb vector is the 1 -form $\eta: \operatorname{Sym}(2) \rightarrow \mathbb{R}$ :

$$
\eta(\Gamma(x, y, z))=B=\frac{x+z}{2}=\frac{1}{2} \operatorname{Tr} \Gamma .
$$


Expressing these objects in a differential-geometry manner:

$$
\xi=\frac{\partial}{\partial x}+\frac{\partial}{\partial z}, \quad \eta=\frac{1}{2}(d x+d z)
$$

it results that $\eta$ is an exact form and hence $C r$ is a cosymplectic structure on $\operatorname{Sym}(2)$. We point out that the usual almost contact structure of the Heisenberg group is Sasakian, [4, p. 42-43].

A more simple transformation on $\operatorname{Sym}(2)$ is provided by the Pauli matrix $\sigma_{3}$ :

$$
\sigma_{3} \cdot \Gamma(a, b, c) \cdot \sigma_{3}=\left(\begin{array}{cc}
a & -b \\
-b & c
\end{array}\right) \in \operatorname{Sym}(2), \quad A\left(\sigma_{3} \cdot \Gamma \cdot \sigma_{3}\right)=\overline{A(\Gamma)}, \quad B\left(\sigma_{3} \cdot \Gamma \cdot \sigma_{3}\right)=B(\Gamma) .
$$

The last method to obtain new symmetric matrices from old symmetric matrices is through the Adjoint representation of $S O(n)$ on $\mathfrak{m}$ :

$$
A d: S O(n) \rightarrow G L(\mathfrak{m}), g \rightarrow A d_{g}: \Gamma \in \operatorname{Sym}(n) \rightarrow A_{g}(\Gamma):=g \Gamma g^{-1} \in \operatorname{Sym}(n) .
$$

For $n=2, \Gamma=\Gamma(a, b, c)$ and $g$ expressed as usually:

$$
g=g(t)=\left(\begin{array}{cc}
\cos t & -\sin t \\
\sin t & \cos t
\end{array}\right)=\cos t \cdot \sigma_{0}-i \sin t \cdot \sigma_{2}=\cos t \cdot I_{2}+\sin t \cdot J, \quad t \in \mathbb{R}
$$

we get that $A d_{g}$ is an orthogonal operator with respect to $<\cdot, \cdot>_{S y m(2)}$ :

$$
\left\{\begin{array}{l}
A d_{g(t)}(\Gamma)=\left(\begin{array}{cc}
a \cos ^{2} t-b \sin 2 t+c \sin ^{2} t & \frac{a-c}{2} \sin 2 t+b \cos 2 t \\
\frac{a-c}{2} \sin 2 t+b \cos 2 t & a \sin ^{2} t+b \sin 2 t+c \cos ^{2} t
\end{array}\right) \in \operatorname{Sym}(2), \\
<A d_{g(t)}\left(\Gamma_{1}\right), A d_{g(t)}\left(\Gamma_{2}\right)>_{\operatorname{Sym}(2)}=<\Gamma_{1}, \Gamma_{2}>_{S y m(2)}
\end{array}\right.
$$

and its Hermitian parameters are:

$$
\left\{\begin{array}{l}
A\left(A d_{g(t)}(\Gamma)\right)=\left[\frac{a-c}{2} \cos 2 t-b \sin 2 t\right]-i\left[\frac{a-c}{2} \sin 2 t+b \cos 2 t\right]=\frac{a-c}{2} e^{i(-2 t)}-b e^{i\left(\frac{\pi}{2}-2 t\right)}, \\
B\left(A d_{g(t)}(\Gamma)\right)=\frac{a+c}{2}=B(\Gamma)
\end{array}\right.
$$

and hence $B$ is a (real) invariant of the Ad representation. For fixed $\Gamma \in \operatorname{Sym}(2)$ and $t=\frac{u}{2}$ of (4.14) we have exactly the diagonal form of $\Gamma$ :

$$
\operatorname{Ad}_{g\left(\frac{u}{2}\right)}(\Gamma)=\operatorname{diag}\left(\lambda_{1}(\Gamma), \lambda_{2}(\Gamma)\right)
$$

For example:

$$
A d_{g(t)}(R)=\left(\begin{array}{cc}
-\sin 2 t & \cos 2 t \\
\cos 2 t & \sin 2 t
\end{array}\right)=R_{e^{i\left(t+\frac{\pi}{4}\right)}} \in O^{-1} \cap \operatorname{sl}(2, \mathbb{R})
$$

\section{Acknowledgments}

We thank Professor Dr. Vladimir Balan (Politehnica University of Bucureşti), Professor Dr. Cornelia-Livia Bejan (Technical University of Iaşi) and especially the editor Bogdan D. Suceavă (California State University at Fullerton) and two anonymous referees for providing very useful remarks.

\section{References}

[1] Bejancu, A.: Groupes de Lie-Banach et espaces localement symétriques. An. Stiint. Univ. Al. I. Cuza Iasi, Sect. Ia. 18, 401-405 (1972).

[2] Bejancu, A.: Sur l'existence d'une structure de groupe de Lie-Banach local. C. R. Acad. Sci., Paris, Sér. A. 276, 61-64 (1973).

[3] Bos, L., Slawinski, M. A.: Proof of validity of first-order seismic traveltime estimates. GEM. Int. J. Geomath. 2(2), 255-263 (2011).

[4] Crasmareanu, M.: Adapted metrics and Webster curvature on three classes of 3-dimensional geometries. Int. Electron. J. Geom. 7(2), 37-46 (2014).

[5] Crasmareanu, M.: A complex approach to the gradient-type deformation of conics. Bull. Transilv. Univ. Braşov, Ser. III, Math. Inform. Phys. 10(59), 59-62 (2017).

[6] Crasmareanu, M.: Conics from symmetric Pythagorean triple preserving matrices. Int. Electron. J. Geom. 12(1), 85-92 (2019).

[7] Crasmareanu, M.: Clifford product of cycles in EPH geometries and EPH-square of elliptic curves. An. Stiint. Univ. Al. I. Cuza Iasi Mat. 66(1), $147-160$ (2020). 
[8] Crasmareanu, M.: Magic conics, their integer points and complementary ellipses. An. Stiint. Univ. Al. I. Cuza Iasi Mat. in press.

[9] Crasmareanu, M., Plugariu, A.: New aspects on square roots of a real $2 \times 2$ matrix and their geometric applications. Math. Sci. Appl. E-Notes 6(1), 37-42 (2018).

[10] González, M. O.: Classical complex analysis. Monographs and Textbooks in Pure and Applied Mathematics, 151. Marcel Dekker, Inc., New York, 1992.

[11] Jensen, G. R., Musso, E., Nicolodi L.: Surfaces in classical geometries. A treatment by moving frames. Universitext. Springer, Cham, 2016.

[12] Lee, J. M.: Introduction to Riemannian manifolds. Second edition. Graduate Texts in Mathematics, 176. Springer, Cham, 2018.

[13] Nicolaescu, L. I.: Morse theory on Grassmannians. An. Ştiinţ. Univ. Al. I. Cuza Iaşi, Mat. 40(1), 25-46 (1994).

[14] Nomizu, K., Pinkall, U.: Lorentzian geometry for $2 \times 2$ real matrices. Linear Multilinear Algebra 28(4), 207-212 (1990).

[15] Özdemir, F., Crasmareanu, M.: Geometrical objects associated to a substructure. Turk. J. Math. 35(4), 717-728 (2011).

[16] Rosenberg, S.: The Laplacian on a Riemannian manifold. An introduction to analysis on manifolds. London Mathematical Society Student Texts, 31. Cambridge University Press, Cambridge, 1997.

[17] Wilkinson, A.: What are Lyapunov exponents, and why are they interesting? Bull. Am. Math. Soc. New Ser. 54(1) 79-105 (2017).

\section{Affiliations}

Mircea CRasmareanu

ADDRESS: University "Al. I. Cuza", Faculty of Mathematics, 700506, Iasi-Romania.

E-MAIL: mcrasm@uaic.ro

http:/ / www.math.uaic.ro/ mcrasm

ORCID ID: 0000-0002-5230-2751 